\title{
Lo vivo en la era neoliberal: la OCDE y el desarrollo de la bioeconomía en México*
}

\author{
Life in a Neoliberal Era: The OECD and the Development of Bioeconomy in Mexico
}

Abigail Nieves Delgado ${ }^{\dagger}$

\begin{abstract}
Resumen
La bioeconomía es un modelo económico que promueve el uso de la biotecnología para la creación de bienes que permitan resolver los problemas sociales y ambientales contemporáneos. La OCDE ha tomado a la bioeconomía como centro de su programa para el futuro. Recientemente en México sucedieron dos eventos importantes en el desarrollo de la biotecnologia: la aprobación de la Ley de Semillas y la construcción del INMEGEN. Ambos forman parte de una agenda nacional para desarrollar la economía del conocimiento en México. A partir del análisis de videos y documentos es posible ver que esta agenda es resultado de la influencia de organismos internacionales como la OCDE. Poner a la OCDE dentro del cuadro ayuda a entender por qué el incumplimiento de las promesas biotecnológicas no perjudican el futuro de la bioeconomía. Por esto, es importante considerar a la OCDE como factor influyente en el desarrollo de la biotecnología y la genómica en México.
\end{abstract}

Palabras clave: bioeconomía - OCDE - INMEGEN - tecnosocial - neoliberal

\begin{abstract}
The "bioeconomy" is a model of economic growth based on biotechnological development. In recent years, the OECD has recommended bioeconomy as a way to solve environmental, health and social problems. It is documented that the OECD has the means to influence decision-making processes in its member countries. Recently, the development of biotechnology in Mexico registered two important events that are the passing of the Seed Law and the construction of INMEGEN. Both belong to a national agenda to inscribe Mexico into the economy of knowledge. Through the analysis of videos and documents it is possible to trace the influence of the OECD in these events. The analysis helps us to understand why unfulfilled promises cause no damage to the bioeconomy project. Thus, it is fundamental to consider the effect of transnational organisms when looking at the national development of biotechnology and genomics.
\end{abstract}

Keywords: bioeconomy - OECD - INMEGEN - technosocial - neoliberal

\footnotetext{
* Recibido: 12 de Mayo de 2014. Aceptado en versión revisada: 20 de Julio de 2014.

+ Doctoranda del Posgrado en Filosofía de la Ciencia, UNAM, México. Para contactar a la autora, por favor, escribir a: abigailnievesdelgado@gmail.com.

Metatheoria 5(1)(2014): 187-202. ISSN 1853-2322.

(c) Editorial de la Universidad Nacional de Tres de Febrero. Publicado en la República Argentina.
} 


\section{Introducción}

En febrero del 2011 apareció una nota periodística en La Jornada donde Julio Frenk, ex secretario de Salud de México, hablaba de la necesidad de la inversión económica en ciencia. "Es fundamental [...] que se entienda que la inversión en la ciencia no es un lujo, sino la base del desarrollo económico en el siglo 21" (Cruz Martínez 2013). Y continuó:

[S]erá un esfuerzo similar al que en 1999 se hizo con el auspicio de la Fundación Mexicana para la Salud para dar sustento científico al proyecto de creación del Instituto Nacional de Medicina Genómica (INMEGEN). Con este organismo [...] México se encuentra en el grupo de naciones que cuenta con información genómica específica de su población y amplias posibilidades de insertarse en una nueva etapa, la de la bioeconomía (Cruz Martínez 2013).

Guillermo Soberón, ex rector de la Universidad Nacional Autónoma de México (UNAM) por su parte señaló que la labor del consorcio promotor del INMEGEN fue evaluar los costos que "tendría para el país retrasar el desarrollo de la medicina genómica" (Cruz Martínez 2013). Tras este análisis "se vio que había el riesgo de que hegemonías exteriores aprovecharan este potencial, así como que el país quedara excluido de la posibilidad de contar con herramientas para el diagnóstico, prevención y predicción de enfermedades" (Cruz Martínez 2013). Después de la primera década de medicina genómica mexicana "los mexicanos tienen la posibilidad de que un futuro (sic) tendrán acceso a métodos de predicción de enfermedades graves como la diabetes, el sobrepeso y la obesidad e incluso el cáncer" (Cruz Martínez 2013). Por esta razón, se debe avanzar hacia la vinculación de la genómica y la bioeconomía. Frenk, Soberón y Gerardo Jiménez Sánchez fueron parte del Consorcio Promotor del INMEGEN creado en el 2001 y de la creación de este instituto en el 2004. La construcción del INMEGEN y el proyecto que abanderó durante sus primeros años de funcionamiento provocaron una reacción importante entre varios grupos de académicos y críticos. Estas reacciones apuntaron a señalar diversos factores que restaban credibilidad al proyecto y al tipo de investigación que se buscaba realizar (por ejemplo López Beltrán 2011). El instituto ha seguido funcionando. Aunque los resultados prometidos hace diez años no se han materializado, el proyecto biotecnológico y genómico en México no ha sido puesto en cuestión sino que ha tomado una dimensión mayor. Recientemente la Universidad Autónoma de Nuevo León auspició el congreso internacional BioUANL 2014 Towards a National Bioeconomy Project in Mexico. Igualmente, en los últimos años la Organización para la Cooperación y el Desarrollo Económicos (OCDE) ha propuesto a la bioeconomía como un modelo económico que resolverá los problemas ambientales, de salud, energía y abasto alimentario mediante el uso de la biotecnología. Si la genómica ha fallado en proveer los resultados concretos que promete ipor qué el proyecto bioeconómico ha ganado terreno no solo a nivel nacional sino internacional? Más aún, ipor qué se favorece, frente a otras alternativas, una respuesta biotecnológica a los problemas sociales y ambientales que se enfrentan en el país?

En este artículo se argumenta que el desarrollo de la investigación genómica en México responde a la influencia de factores internacionales, como los lineamientos de la OCDE y no a su capacidad de ofrecer beneficios tecnológicos específicos para la población mexicana o su economía, como generalmente se ha planteado. Es decir, el desarrollo de la genómica como parte integral del modelo de bioeconomía hace posible que México se integre al modelo neoliberal internacional, y no tiene mucho que ver con el desarrollo de fármacos, o soluciones biotecnológicas agrícolas, energéticas, etc. Por esta razón, la falta de resultados concretos no perjudica el proyecto genómico. Situar el proyecto bioeconómico mexicano dentro de un contexto internacional ofrece algunas respuestas. Pone en claro que la inversión en bioeconomía tiene el objetivo de generar las condiciones para expandir y desarrollar el mismo modelo económico al que pertenece y que los resultados tecnológicos concretos no necesariamente son el motor principal de la inversión en biotecnología. Además explica por qué proyectos como el INMEGEN fueron financiados en décadas pasadas. 
Para mostrar que hay una influencia importante de la OCDE en el proyecto mexicano de bioeconomía se analizarán los videos institucionales del INMEGEN y algunas conferencias internacionales de bioeconomía y genómica. Con este análisis se busca identificar qué elementos retóricos y visuales se han movilizado para justificar y promover la inversión económica en la genómica y la bioeconomía. En este análisis es posible ver que hay elementos recurrentes que además no aparecen sólo en el contexto mexicano, sino se pueden encontrar también en otros contextos. Esta recurrencia nos hace entender que el proyecto genómico y bioeconómico mexicano forma parte de una tendencia internacional hacia la bioeconomía, que a su vez es parte de un plan económico transnacional. Como se verá, una consecuencia de esta visión genómica de los problemas ambientales, de salud, alimentarios, etc., es que éstos no pueden ser resueltos desde otros frentes. Hay una performatividad en la perspectiva bioeconómica. Es decir, el poner en términos biotecnológicos los problemas que se busca resolver, implica la puesta en marcha del proyecto biotecnológico para resolverlos.

Este artículo tiene tres partes. En la primera parte se introduce a la bioeconomía junto con algunas de sus características principales. Además se esboza el contexto histórico en el que esta nueva economía se ha generado dentro de una perspectiva internacional. En la segunda parte se presenta un elemento fundamental de la bioeconomía. Este es la "visión genética de la vida." Se habla entonces de cómo el "mito de lo genético" ha permeado en diferentes ámbitos y ha facilitado que la bioeconomía se perciba como un proyecto factible. En la tercera sección se lleva la discusión hacia el terreno mexicano. Se rastrea la influencia de la OCDE en las decisiones políticas recientes. A través del análisis de diversos documentos y videos se muestra que el desarrollo de la genómica en México está supeditado a un plan internacional que apunta hacia la bioeconomía.

\section{La OCDE y la bioeconomía}

La Organización para la Cooperación Económica Europea (OECE) se formó en 1948 con la finalidad de administrar la ayuda a los países europeos provista por los Estados Unidos y Canadá tras la Segunda Guerra Mundial. En 1961 la OCDE remplazó a esta organización. Desde entonces ha tenido como objetivo "construir economías fuertes [...] mejorar la eficiencia [...] expandir el libre comercio y contribuir al desarrollo en países industrializados y en desarrollo" (OECD 2015a). A partir de 1992, la OCDE se ha abierto a la participación de países no miembros de la organización. En 1992 Corea del Sur fue el segundo país asiático en pertenecer a la OCDE y México fue aceptado en 1994, después de haber firmado el Tratado de Libre Comercio con Estados Unidos y Canadá. La organización también ha sido clave en la consolidación de la alianza del Atlántico Norte en oposición al bloque soviético. Tras su desintegración, varias organizaciones internacionales, entre ellas la OCDE "facilitaron" la transición de los estados de Europa del Este hacia una economía de mercado (Mahon \& McBride 2009, p. 92). Aunque es una organización principalmente formada por países europeos, la influencia de los Estados Unidos en la dirección y agenda de la organización es algo que ha sido subrayado. Los Estados Unidos son el principal contribuyente económico de la organización, seguido de Japón. Hasta la mitad de los años setenta la OCDE funcionaba siguiendo un enfoque Keynesiano (Mahon \& McBride 2009, p. 94). Actualmente la organización promueve un enfoque neoliberal, aunque dentro de la OCDE hay cierta posibilidad de diálogo entre diferentes ramas de la organización haciendo posible diferentes expresiones y corrientes ideológicas (Mahon \& McBride 2009, p. 98). No obstante, estas expresiones nunca son totalmente ajenas al paradigma contemporáneo centrado en la economía de mercado.

La OCDE posee características que le permiten regular y estandarizar las decisiones políticas de los diferentes estados sin de hecho tener el poder de interferir en estos. Forma parte de una nueva red de organizaciones internacionales que ejercen un tipo de "gobernanza sin gobierno" (Mahon \& McBride 2009, p. 86). Este sistema emergente de gobernanza transnacional posee medios de regulación inquisitivos y mediativos que permiten homogeneizar los problemas a resolver, las preguntas que se hacen respecto a estos problemas, el vocabulario con el que se les denota y los medios con que se 
discuten y resuelven. Es un ejercicio de estandarización y diseminación de normas y estándares de comparación que permite evaluar el desempeño de los países uno frente a otro respecto a parámetros específicos. Mientras la regulación inquisitiva tiene que ver con la vigilancia y el monitoreo de los estados, la regulación mediativa se refiere principalmente a establecer marcos compartidos para la discusión de expertos respecto a un problema y sus soluciones. Ambos procesos contribuyen en el monitoreo y evaluación de los países en relación a los estándares que la misma organización ha establecido (Mahon \& McBride 2009). Mediante la discusión de expertos, la OCDE se encarga de proponer lineamientos a seguir. Los gobiernos no están obligados a seguir estar recomendaciones, sin embargo, hay evidencia de que en muchos casos la OCDE ha sido capaz de influir en las políticas públicas adoptadas en algunos países (Mahon \& McBride 2008, 2009, Salzman 2000). En los últimos años la OCDE ha presentado un modelo económico fundamentado en el uso de la biotecnología para resolver problemas ambientales, de salud, energía y abasto alimentario. Este modelo lleva por nombre bioeconomía.

\subsection{La bioeconomía}

Melinda Cooper (2008) rastrea el fenómeno de la bioeconomía hasta la "revolución biotecnológica" de la época de Reagan en los Estado Unidos. Para ella, la bioeconomía es el resultado del cruce entre las teorías neoliberales de crecimiento y crisis con la especulación en el futuro de las ciencias de la vida y las posibilidades reproductivas y regenerativas de los organismos (Cooper 2008, p. 11). Las previsiones fatalistas del Club de Roma en su reporte The Limits to Growth de 1972 presentaron una visión del futuro donde el modelo fordista de desarrollo ponía en juego la posibilidad de la vida en la tierra. Este reporte puso en la mesa de discusión la idea de la finitud de los recursos de la tierra y como consecuencia, los límites del crecimiento (Cooper 2008, p. 16). En el contexto de los Estados Unidos, una respuesta desde la derecha fue llamar a una "restructuración radical de la economía estadounidense" (Cooper 2008, p. 17). Se argumentó que para que los Estados Unidos pudieran conservar su dominancia económica, necesitarían moverse de una economía industrial a una economía basada en la innovación. Esta economía post industrial además de responder a las necesidades de crecimiento económico, ofrecía una solución a los problemas ambientales presentados por el Club de Roma. Según los teóricos de la época de Reagan, la biotecnología representaba una forma de internalizar y superar los límites del crecimiento (Cooper 2008, p. 18). Cooper afirma que "[1]a revolución biotecnológica, es el resultado de una serie de medidas legislativas y regulatorias diseñadas para reubicar la producción económica a los niveles genético, microbiano y celular, así que la vida se anexa, literalmente a los procesos de acumulación capitalista” (Cooper 2008, p. 19).

De igual forma, Vincenzo Pavone (2012) identifica una relación mutuamente constitutiva entre la bioeconomía y el neoliberalismo. De acuerdo a su análisis, durante la década de los ochenta como resultado del colapso de los acuerdos de Bretton Woods y las crisis del petróleo de 1973 y 1979, el neoliberalismo comenzó a tener fuerza en la agenda política sustituyendo la construcción de un estado social por la privatización de los servicios. La primera fase de este cambio político sería conocida como Roll Back. Sin embargo, no sería sino hasta un segundo momento conocido como Roll Out en donde se dejaría de lado el modelo fordista de desarrollo y se perseguiría un nuevo modelo basado en la innovación en el mercado y la comercialización de los avances científicos y tecnológicos (Pavone 2012, p. 153). Según Pavone, durante la fase de Roll Out se dio un tipo de relación recíproca entre la ideología liberal y el desarrollo de tecnologías donde las nuevas tecnologías configuraron cómo se entendió e implementó el neoliberalismo (Pavone 2012, p. 154).

En el año 2006, la OCDE presentó su proyecto "Bioeconomía al 2030" como parte de su programa de "Futuros Internacionales" buscando "diseñar una agenda bioeconómica para los gobiernos" miembros de la organización (Goven \& Pavone 2015, p. 304). En este informe, la bioeconomía se define como un "mundo" donde las contribuciones de la biotecnología representan una proporción significativa de la producción económica. Ésta además consta de tres elementos: conocimiento biotecnológico, biomasa renovable y la integración entre las aplicaciones (OECD 2009, p. 22). El 
conocimiento biotecnológico se centra en entender y manipular DNA, RNA, proteínas y células, tejidos, órganos y organismos. Se complementa con el desarrollo de la bioinformática para el análisis de genomas y proteínas y se enfoca en el desarrollo de nuevos procesos para producir biofarmacéuticos, vacunas recombinantes, variedades nuevas de animales, plantas y enzimas (OECD 2009, p. 23). Su potencial radica en la producción de tecnología genérica y en la creación de herramientas e invenciones con usos múltiples, lo que ayuda a crear economías de alcance (OECD 2009, p. 23). La OCDE subraya que para que el poder de la bioeconomía pueda ser aprovechado, es necesario que los usuarios, las empresas y los gobiernos participen y colaboren, y que se defina una agenda de políticas que puedan regular esta forma de mercado emergente. Al centro de los intereses de la organización está el "mejoramiento económico y de bienestar de las personas en el mundo" (OECD 2015c). Se debe imaginar un nuevo sistema económico "más humano y digno" donde la "sostenibilidad en los negocios es una necesidad, no una opción". Este modelo capitalista más "humano" debe moverse de un sistema de energía fósil a uno de menor consumo de carbono. Se trata de construir un sistema que nos permita tener nuestro "futuro ambiental" en las manos. Es una economía de compartir, con menos desperdicio y menos posesiones. Una economía que garantiza el futuro al preocuparse por el cuidado del medio ambiente (OECD 2015b). La bioeconomía se presenta como la respuesta a estas demandas. Promete acabar con los problemas generados por el sistema capitalista (pobreza, contaminación, desigualdad) mediante el uso de innovaciones tecnocientíficas. Varios autores han señalado la naturaleza promisoria del futuro tecnosocial de la bioeconomía (Brown \& Michael 2003, Cooper 2008, Mike Fortun 2005, Taylor-Alexander \& Schwartz-Marín 2013). Las promesas tecnocientíficas tienen la capacidad de "generar cambios en el presente, sin importar si éstas o no se cumplen" (Goven \& Pavone 2015, p. 314). Según Joanna Goven y Pavone (2015) esto es un imaginario típico tecnosocial (Goven \& Pavone 2015 , p. 306). Lo interesante de estos imaginarios es que funcionan de manera performativa. El carácter performativo de estas promesas se encarga de "habilitar ciertos mundos tecnocientíficos y deshabilitar otros" (Brown \& Michael 2003, p. 14). Esto es, garantizan los espacios de acción y mercados para su propia supervivencia en el futuro "es una estrategia para proteger y facilitar la acumulación de capital de cara al creciente reconocimiento de la amenaza de la catástrofe ambiental" (Goven \& Pavone 2015, p. 310). Es decir, se proponen medios capitalistas para solucionar problemas que el mismo capitalismo ha generado. El proyecto de bioeconomía de la OCDE propone solucionar estos problemas sociales mediante la producción de objetos biotecnológicos que reviertan los efectos nocivos del consumo capitalista (remediación de espacios contaminados, biocombustibles diferentes al carbono, fármacos contra la obesidad o la hipertensión). Esta solución garantiza el curso capitalista de la economía. Otra consecuencia de esta relación entre el desarrollo de tecnologías y el neoliberalismo, y de la forma en que se entienden los problemas sociales y se busca resolverlos, es la extensión del mercado hacia nuevos territorios. La visión tecnocientífica se expande hacia nuevos espacios humanos como la salud, el medio ambiente y la alimentación humana. Al mismo tiempo que deja fuera otros principios como el bien común, la acción colectiva y la cohesión social (Pavone 2012, p. 158).

El proyecto de bioeconomía propuesto por la OCDE, un organismo regulador y normativo transnacional, podría entenderse como un proyecto político que "induce y facilita ciertas acciones mientras impide otras [...] se trata de dar lugar a un conjunto particular de cambios político institucionales que darán forma a los parámetros de posibles acciones futuras" (Goven \& Pavone 2015, p. 302). Estos mismos autores identifican una línea argumentativa clave que es recurrente en la propuesta bioeconómica. Esta consiste en cuatro argumentos básicos: 1. La regulación estatal actual representan un problema, 2. Porque obstruye el potencial de la bioeconomía y 3. Esto implica que se debe rediseñar las regulaciones. 4. Para evitar el arrepentimiento por frenar el potencial de la bioeconomía (Goven \& Pavone 2015, pp. 305-306). En el análisis siguiente veremos cómo se ponen en juego estos argumentos tanto en los videos como en las conferencias. Con esto se busca mostrar que esta ideología neoliberal promovida por la OCDE ha sido progresivamente insertada en el contexto mexicano a partir de la apertura de instituciones de investigación y aprobación de leyes durante la primera y segunda décadas de los años 2000. 


\subsection{Biotecnología en México: años 2000}

Durante la primera década de los años 2000 se registraron por lo menos dos momentos importantes en el contexto biotecnológico de México. El primero tiene que ver con la agricultura. En el año 2005 se publicó la llamada Ley de Bioseguridad de Organismos Genéticamente Modificados (OGMs), también conocida como Ley Monsanto, y un año después, en el año 2006, se aprobó su complemento, la Ley de Producción, Certificación y Comercio de Semillas. Esta ley se implementó para regular que todas las semillas que se cultiven en el campo mexicano sean "semillas certificadas". Estas semillas certificadas deberían ser semillas de calidad controlada, con mayor resistencia a plagas y enfermedades y que pueden producir cultivos más homogéneos. El Programa Nacional de Semillas instituyó un comité conformado por el sector público y privado a cargo de definir qué productores pueden vender semillas certificadas y quiénes no. La ley también estipula que todo productor debe utilizar semillas certificadas y que debe poseer su recibo de compra por lo menos por dos años, de lo contrario se genera una multa conforme a lo estipulado en la ley. En la ley de semillas no se habla específicamente de semillas modificadas genéticamente, sin embargo su papel en la regulación e institucionalización del mercado, producción y cultivo de semillas producidas mediante técnicas de DNA recombinante y otros procesos biotecnológicos es claro. El día en que la ley fue aprobada el entonces director de investigación de Monsanto, Manuel Oyervides, afirmó que "con el uso de biotecnología y con la nueva Ley de Semillas, la empresa podrá aumentar el capital para la investigación superando los 20 millones de dólares anuales y obteniendo mejoras para los productores nacionales" (Martínez 2007). Para que la Ley de Semillas y la de Bioseguridad entrarán en vigor se debería publicar un reglamento que no fue publicado hasta el 2011 (DOF-Diario Oficial de la Federación 2011). Sin embargo, hubo declaraciones entusiastas ya en el 2007 por parte del presidente del Consejo de Productores de Maíz de Tamaulipas Perfecto Solís quien afirmó que "[c]on ese reglamento o sin él empezaremos a sembrar maíz transgénico y, si es necesarios, recurriremos al uso de la fuerza para defender los cultivos”. En su perspectiva, que los campesinos no contaran con la posibilidad de utilizar OGMs implicaba ponerlos en desventaja con los productores de maíz de los Estados Unidos (Pérez 2007).

Un segundo evento biotecnológico relevante de la década del 2000 es la presentación del mapa genómico de los mexicanos al presidente Felipe Calderón (Herrera Beltrán 2009). Con este evento se habría cumplido con uno de los principales objetivos de la primera etapa de investigación en el INMEGEN. Aunque el evento se trató de la publicación de un artículo científico sobre la composición genética de la población mexicana (Silva-Zolezzi et al. 2009), en los medios se publicitó como "el libro de la vida de los mexicanos" (por ejemplo, Herrera Beltrán 2009). Después de la salida de Gerardo Jiménez Sánchez de la dirección del INMEGEN, el instituto pareció cambiar de rumbo y dejar de lado el proyecto que encabezó la primera etapa. Sin embargo, la idea de que la población mestiza mexicana es especialmente susceptible a la diabetes fue heredada a la Dra. Marta Mejivar Iraeta, investigadora de la Facultad de Química de la UNAM y del INMEGEN, y su "Genoteca Indígena". Los proyectos de genómica médica en México desde su aparición se han justificado a partir de suponer una especificidad biológica de los mexicanos. Y junto a esta especificidad biológica una especificidad patológica que los hace más susceptibles a ciertas enfermedades, como la diabetes. Esta justificación no es exclusiva del caso mexicano y está detrás de la mayoría de los proyectos genómicos en el mundo que se enfocan en poblaciones específicas y asumen que son "únicas," "homogéneas" y que de alguna manera han estado "aisladas" (como en el caso de Islandia) (Fortun 2010, p. 147) o por el contrario "mezcladas" con otras (como en el caso mexicano). La idea general que está detrás es el supuesto de que las poblaciones en el mundo divergen entre sí y por lo tanto es importante estudiarlas (genéticamente). El proyecto del "Mapa Genómico de los Mexicanos" desató numerosas respuestas críticas que hicieron visibles puntos débiles de la investigación y el proyecto en general. Algunos de estos textos se han centrado en criticar el uso de categorías raciales para justificar la investigación biomédica (López Beltrán \& Vergara Silva 2011, Montoya 2011) y han dado claridad a las diferentes acepciones del término "mestizo" en el contexto de las ciencias biológicas (López Beltrán \& García Deister 2013). Otros análisis han planteado la pregunta desde una perspectiva ligeramente diferente. Por ejemplo Samuel Taylor-Alexander y 
Ernesto Schwartz-Marín (2013) se preguntan cómo fue que un proyecto como el INMEGEN pudo ganar respaldo político en un país donde la oferta médica para la población es insuficiente. Un argumento similar se puede hacer respecto al tema de la agricultura. ${ }^{1}$ Tanto para los problemas de salud como para los agrícolas se podrían ofrecer soluciones que no necesariamente implicaran desarrollos biotecnológicos.

En el caso del INMEGEN Taylor y Schwartz (2013) señalan que fue el presentar al instituto como una inversión económicamente redituable lo que ayudó a su materialización. Son sin duda razones económicas las que han contribuido al desarrollo de la biotecnología y la genómica en México. Sin embargo estos enfoques se quedan incompletos cuando no se reconoce como factor fundamental la influencia de la OCDE en las decisiones políticas de los últimos años. Poner dentro del cuadro a la OCDE, y a otras organizaciones internacionales como la Organización de las Naciones Unidas para la Educación, la Ciencia y la Cultura (UNESCO), la Organización Mundial de la Salud (OMS) (SchwartzMarín 2011, p. 132) y el Banco Mundial (DOF-Diario Oficial de la Federación 2014) hace posible ver que en el caso del INMEGEN, este instituto no es un fin, sino un medio. Es decir, que el INMEGEN es una institución que permite que México se integre en la tendencia internacional hacia la bioeconomía promovida en los últimos años por esta organización internacional.

El imaginario tecnosocial puesto en la mesa por el proyecto bioeconómico de la OCDE no podría funcionar sin otro elemento fundamental. Esto es una concepción específica de la vida y del estudio de ésta que se reduce, o por lo menos favorece, al nivel de lo genético. La siguiente sección trata de este aspecto. Como se verá, son estas imágenes de lo vivo las que hacen posible que el proyecto de la bioeconomía goce de credibilidad y prestigio tanto en el ámbito científico como en esferas menos especializadas. Estos elementos visuales aparecen integrados a los argumentos de la bioeconomía en los documentos y videos que posteriormente se analizarán.

\subsection{Imágenes de lo vivo}

En el periodo de la posguerra la influencia de instituciones como la Fundación Rockefeller fue fundamental en el establecimiento de maneras específicas de entender lo vivo. La fundación Rockefeller incentivó la organización científica alrededor de ciertos problemas, metodologías, sistemas experimentales y métodos de investigación específicos. Detrás de esta selección de problemas y fenómenos para "hacer biología" había una concepción de la vida muy precisa. Según Edward Yoxen, en el siglo 20 la mayoría de los científicos profesaban un materialismo primitivo o cuasi materialismo, que sugería que el problema de la vida se localiza en la organización de la estructura de la materia viva (Yoxen 1982, p. 129). El programa de la Rockefeller logró organizar los intereses y los recursos de muchas personas alrededor de un tipo específico de problemas e investigación, a la vez que definió los parámetros de lo que contaba como una explicación en biología (Yoxen 1982, p. 133). Esto sentó las condiciones para que los biólogos moleculares y en general, "la visión molecular de la vida", ganara prestigio y poder en la esfera de la investigación científica. Logró así las institucionalización de "su concepción de vida" (Yoxen 1982, p. 134) Para Lily Kay, sentar el "lugar esencial de la vida" en el nivel molecular logró transformar los "fundamentos epistemológicos de la investigación biológica" (Kay 1993, p. 7). La "visión molecular de la vida" se instaló al centro de un imaginario tecnosocial compuesto por la imaginación científica de la intervención tecnológica en todos los aspectos de lo vivo, desde lo minúsculo hasta lo humano.

A finales de los años cincuenta, comenzaron a aparecer programas en la televisión británica que mostraban esta nueva forma de hacer biología. What is life? uno de los primeros, fue presentado el 1 de diciembre de 1959 con el argumento principal presentar a los nuevos biólogos, jóvenes investigadores enfrentándose a una "nueva redefinición de la vida" mediante su trabajo en biología celular y genética (Yoxen 1982, p. 139). Desde entonces, la doble hélice del DNA continúa apareciendo en numerosas publicaciones e imágenes especializadas o no. La movilización de "soporte cultural" desde estas

${ }^{1}$ El caso de la agricultura no se puede tocar aquí por cuestiones de espacio. 
primeras apariciones hasta sus más recientes versiones han contribuido fundamentalmente en la consolidación de este programa de investigación (Yoxen 1982, p. 139).

El "descubrimiento" de la doble hélice de DNA y su importancia como pieza fundadora de un campo de investigación ha sido considerado como un "logro heroico" (Yoxen 1985, p. 164) por los miembros de su disciplina y la historiografía más ortodoxa. Bastaron tan solo 21 años después de tal descubrimiento para que en 1974 la BBC realizara una película con los dos científicos principales Watson y Crick, y para que The Times publicará un artículo sobre biología molecular. Nature por su parte, publicó un suplemento especial con una portada donde aparece una caricatura con dos hombres deslizándose de una hélice (Yoxen 1982, p. 164). Posteriormente, esta publicación ha conmemorado los 30, 50 y 60 años del gran descubrimiento. La imagen de la doble hélice ha aparecido en incontables publicaciones científicas, escolares, de divulgación, comerciales, etc., y ha significado también diversas cosas (como genética, identidad, individualidad, constitución o make up, naturaleza, inmutabilidad), legibles en una u otra acepción.

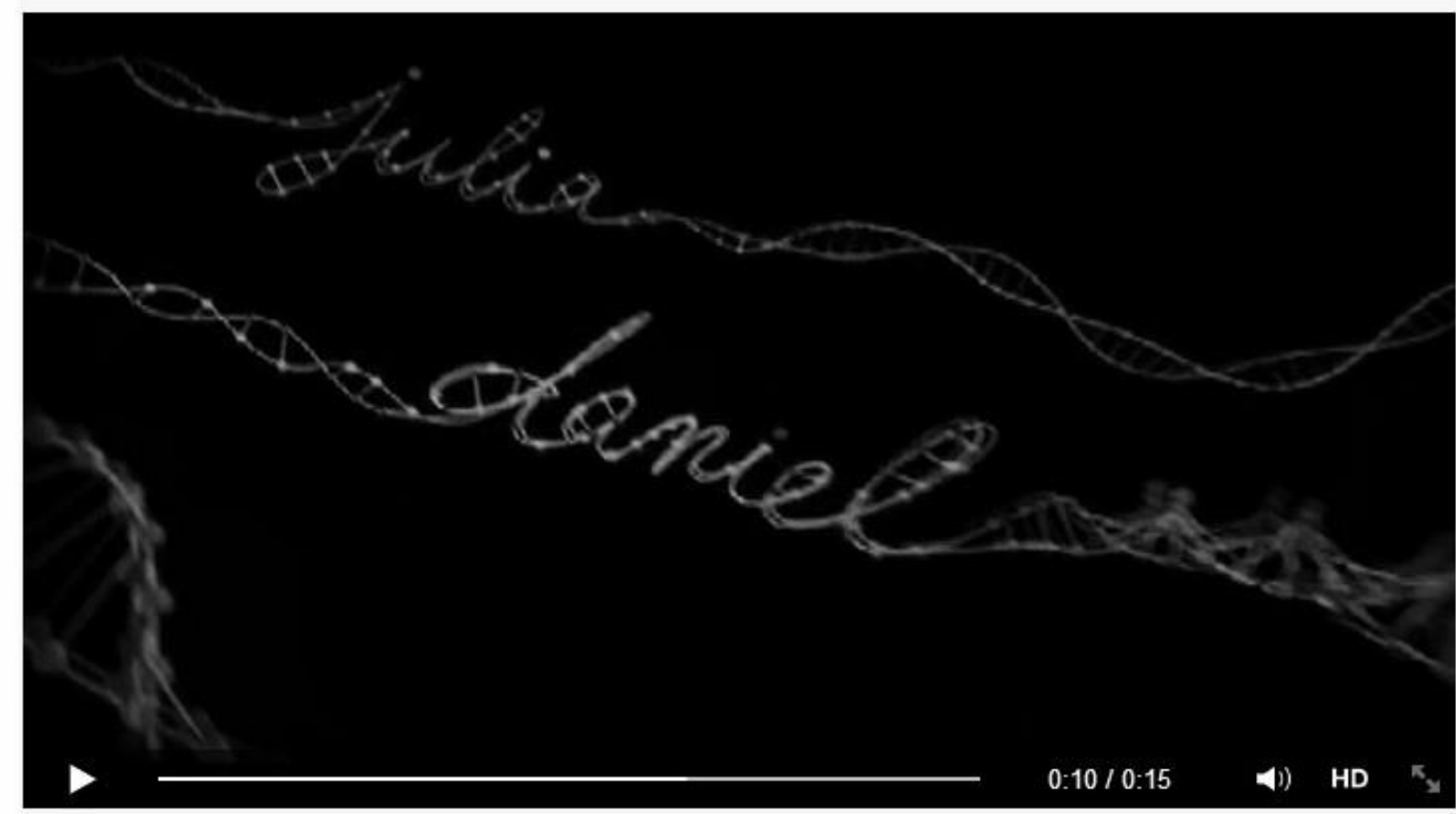

Foto 1. Captura de pantalla del video American Express: "[E]l DNA es lo que te hace ser tú. Un individuo con necesidades individuales, que siempre estamos encantado de atender. Está en nuestra constitución”2 (American Express. Realize the potential 2011).

A continuación se presentarán algunos extractos de los videos del INMEGEN del 2009 y 2012 que están disponibles en Youtube y en el sitio oficial del instituto. Con el análisis de estos videos se muestra que hay una recurrencia de elementos clave en la argumentación en bioeconomía y la genómica. Podemos ver cómo imágenes científicas y argumentos económicos se movilizan de manera conjunta para presentarnos la posibilidad de un futuro tecnosocial lleno de posibilidades y esperanza. Tal y como señalan Brown y Michael (2013) la retórica performativa de la bioeconomía se encarga de habilitar los escenarios tecnocientíficos que convienen para su propia acción. Es decir, genera en el presente el futuro que permita garantizar la acumulación de capital mediante sus propias promesas tecnocientíficas. En los videos podemos ver como las cadenas de DNA se mezclan con argumentos

\footnotetext{
2 Traducción de "[D]NA is what makes you, you. An individual with individual needs, which we are always delighted to help with. It's in our make-up. American Express, Realize the potential".
} 
sobre la especificidad genética de los problemas de salud a resolver y el posible riesgo que conlleva no actuar ahora para propiciar el desarrollo de la bioeconomía.

\section{La bioeconomía en imágenes: INMEGEN 2009}

El video inductivo del INMEGEN (Villacorta 2009), que está disponible en Youtube por lo menos desde el 2009, comienza con la imagen de una célula computarizada en cuyo núcleo vemos a los cromosomas separándose hacia los dos extremos con ayuda del huso acromático. Inmediatamente después, en un movimiento rápido, aparecen los cromosomas por separado flotando en un espacio azul para dar paso entonces a la imagen de la doble hélice, mientras el narrador explica en el segundo 0:05 del video que el genoma humano es "quizá uno de los descubrimientos científicos más importantes para la humanidad". Entonces aparece la imagen del INMEGEN que nos dicen es la institución mexicana que se encarga del estudio del genoma humano y que coloca a la vanguardia de la investigación médica a México. Después nos explican qué es el genoma humano y cómo es que este DNA es responsable de nuestra constitución, cuerpo, individualidad, dentro de un espacio digital verde similar al de la película Matrix.

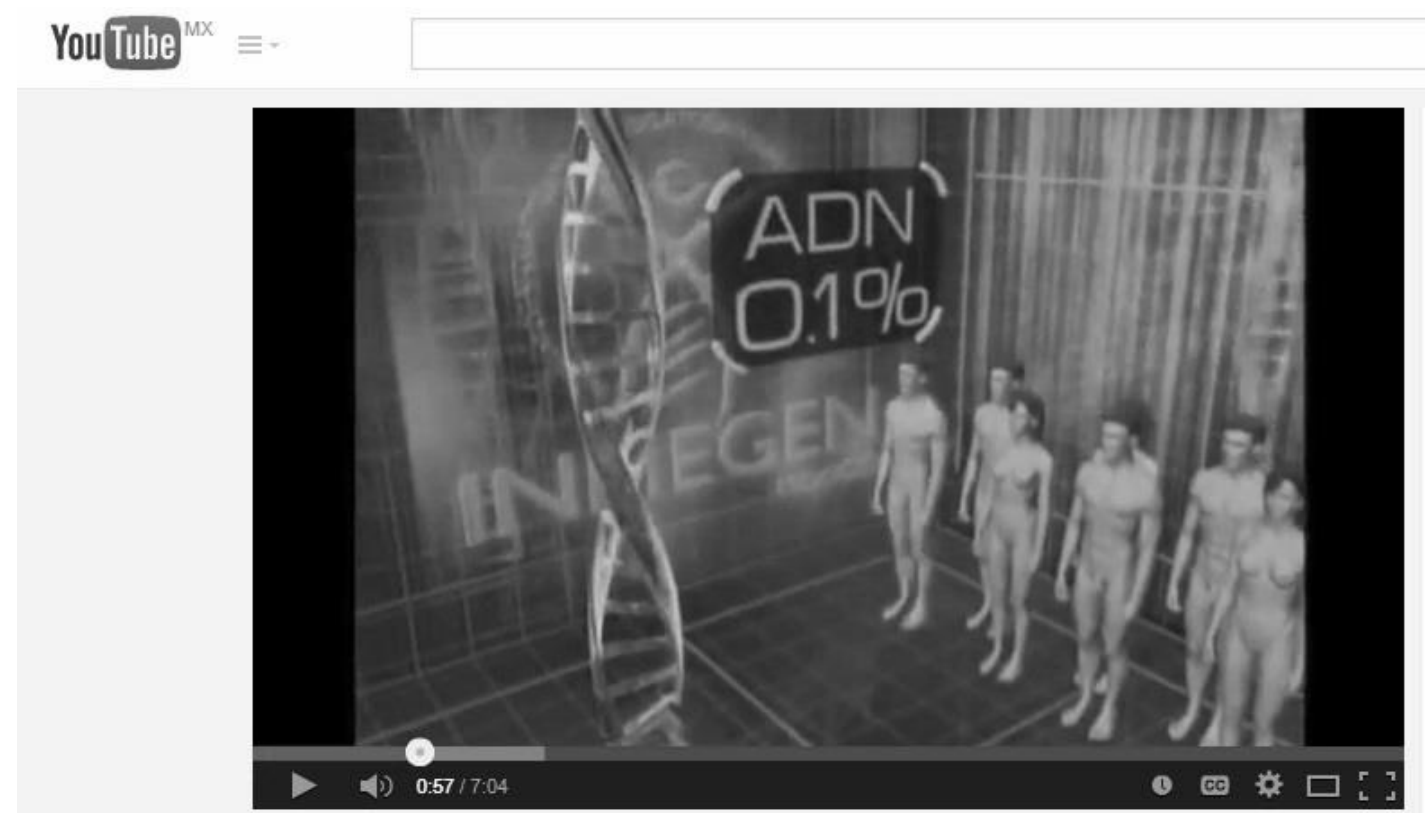

Foto 2. Captura de pantalla del video institucional del INMEGEN 2009.

Una vez terminada la parte central sobre los "hechos científicos" el video se mueve hacia explicar la relevancia de la investigación genómica en específico para la población mexicana: "Cada población tiene sus particularidades genómicas y México no es la excepción. Ya que la mayor parte de su población es producto de un mestizaje entre poblaciones de Mesoamérica y españoles por ello resulta indispensable que México desarrolle investigación médica en medicina genómica que beneficie a la salud de los mexicanos. Para esto se creó el INMEGEN" (1'49").

Finalmente el video se mueve hacia la justificación económica. El primer argumento es que, la investigación genómica de las enfermedades comunes de una población reducirá el costo de su tratamiento (4'30"). Entonces se exponen los "otros alcances" de la medicina genómica. Entre estos alcances están la generación de productos y servicios, formas de comercio, comunicación y organización social que no son especificadas pero que hacen de la medicina genómica represente "oportunidades de desarrollo en este nuevo orden de la economía basada en el conocimiento. Oportunidades que dejarán huella sin precedente en la medicina a nivel nacional e internacional" (5'45”). La economía basada en el conocimiento es lo que después será conocido como bioeconomía. Casi llegando al minuto 6 aparece el Dr. Lee Hartwell quien en 2001 ganó el premio Nobel de 
medicina por sus investigaciones sobre el ciclo celular. Él nos dice: "El impacto que tendrá el Instituto de Genómica debería ser muy grande. La industria y la biotecnología, las compañías farmacéuticas, y la gente que está formando empresas y todo lo que tenga que ver con el negocio de la vida o de la salud, requerirá entender la biología molecular" (5'59"). Inmediatamente después el narrador nos dice que apoyar a la investigación del INMEGEN nos traerá grandes beneficios a la población mexicana y después reitera: "contribuir al INMEGEN es vital para el desarrollo de México pues además de mejorar la calidad de vida de su población permitirá una mayor productividad y por ende competitividad a la economía mexicana" (6’35”).

En este video producido por el patronato fundador del INMEGEN se hacen explícitas las dos promesas principales de esta institución: la salud de la población mexicana y se podría decir, la salud de la economía mexicana. La breve mención de la "economía del conocimiento" se hará mucho más explícita en el video institucional del INMEGEN del 2012. A la fecha, no hay productos específicos, estadísticas o informes sobre cómo, cuánto y de qué manera ha influido el INMEGEN en mejorar la calidad de vida de la población en México. Esto tampoco se aclara en el video del 2012. Por el contrario, en éste se intensifica la urgencia y el llamado a aumentar la inversión en la ciencia genómica.

\subsection{La bioeconomía en imágenes: INMEGEN 2012}

El video institucional del INMEGEN del 2012, alojado en su sitio de internet desde hace tres años, tiene una estructura similar al del 2009. Como en el anterior, primero habla sobre "hechos científicos innegables" como que "el genoma es el descubrimiento más grande de la historia de la humanidad". Al mismo tiempo, se muestran imágenes de los cromosomas y del DNA que, a diferencia del video del 2009, pretenden representar a los objetos genéticos mediante una tecnología de visualización microscópica más sofisticada. La cadena de DNA y los cromosomas aparecen con colores de contraste y volumen que intercalados con imágenes de microscopia parecen dotados de un realismo que en la versión anterior no se pretendía.

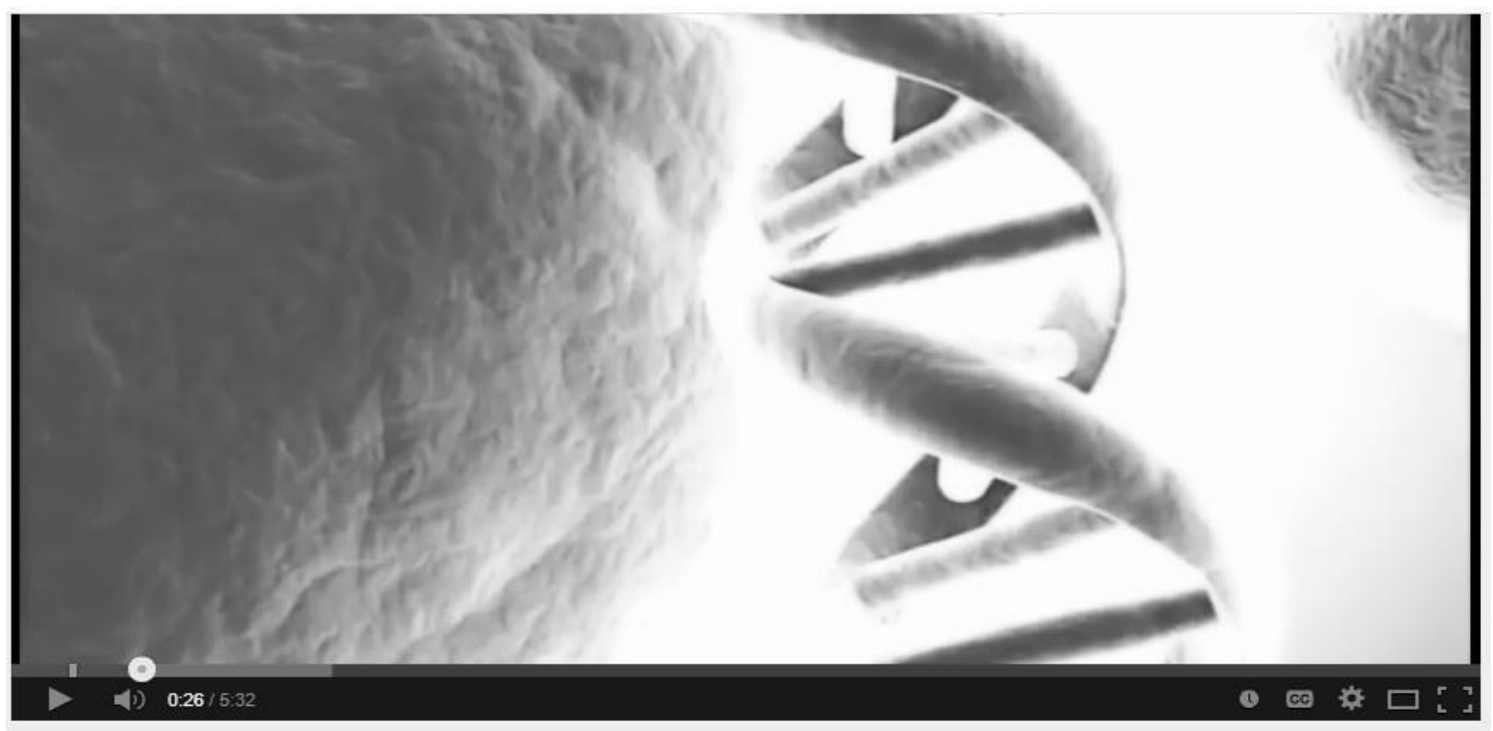

\section{Video Institucional INMEGEN}

Foto 3. Captura de pantalla del video institucional del INMEGEN 2012.

Después de establecer los hechos biológicos fundamentales de la constitución humana, una explicación idéntica a la de la versión anterior, el video se mueve a explicar a detalle la composición del DNA: "[q]ue contiene cerca de tres mil doscientos millones $(3,200,000,000)$ de letras genéticas que conocemos por sus iniciales AGTC" (0'39"). Explicar que el DNA está conformado por letras va a permitir que se introduzca la noción de Polimorfismo de Nucleótido Simple (SNP) y se pueda explicar con este recurso la diferencia que hay entre el genoma de diferentes poblaciones humanas: "Cuando 
algunas personas tienen en una posición de la cadena una $\mathrm{T}$ y otras personas esa misma posición tienen una A se le conoce como polimorfismo de un solo nucleótido o SNP. Se tienen ya catalogadas más de 10 millones de SNPs que demuestran la diversidad genética del género humano" (1'04").

En este video desaparece el énfasis en la especificidad genética de la población mexicana como resultado del proceso de mestizaje. Sin embargo esto no quiere decir que la investigación del INMEGEN no dependa de la genética poblacional. En el sitio de INMEGEN también se aloja un video que explica qué es la genética poblacional y que si bien presenta una versión más compleja del proceso de mestizaje, termina por describir un panorama conocido. ${ }^{3}$

El cambio más interesante para el tema de este artículo está en la notable presencia de la OCDE que no era mencionada en el anterior:

\begin{abstract}
Actualmente de acuerdo a datos de la OCDE, México tiene la inversión más baja en ciencia tecnología e innovación. Es necesario cambiar dicho panorama y solo se conseguirá creando sinergias entre el sector público y privado. Si se logra consolidar una adecuada inversión en ciencia y tecnología se obtendrá una economía más sostenible, basada en la competitividad industrial, la productividad, el empleo y el nivel de vida de los mexicanos. El presente es una oportunidad que dejará huellas sin precedente en el mundo de la medicina a nivel nacional e internacional. Un esfuerzo que sin duda es un beneficio trascendental para los mexicanos, un compromiso decidido con el desarrollo institucional de la medicina, un logro que permitirá la atención de enfermedades comunes, avances con tecnología de punta para lograr el desarrollo pleno de nuestra gran nación. Así es el instituto nacional de medicina genómica de México, INMEGEN (4’23”)
\end{abstract}

Como se verá en la siguiente sección, a partir del año 2012, habrá dos argumentos consecutivos en relación a la inversión económica en genómica. En primer lugar se establecerá una comparación de la economía mexicana con otras economías emergentes. En esta comparación se subraya que otras economías emergentes han logrado mejorar su condición económica al invertir en ciencia y tecnología. El segundo argumento, que aparece más tarde en el 2013 muestra con números claros las ganancias concretas que la bioeconomía ha generado en países donde se le ha implementado. En la siguiente sección se presenta cómo se han desarrollado estos argumentos.

\title{
3.2. La bioeconomía en palabras: "Better policies for better lives"
}

En la "2a Feria de Innovación Tecnológica de la Ciudad de México. Generando riqueza a partir del conocimiento", Gerardo Jiménez Sánchez es entrevistado después de su conferencia. Bertha Alicia Galindo le pregunta ¿Qué se puede hacer? Él entonces responde que

Con el genoma humano podemos ya identificar variaciones que nos predisponen a enfermedades comunes como diabetes hipertensión o cáncer o variaciones genéticas que nos predisponen a tener efectos adversos a medicamentos de uso común. Estos son avances concretos de la medicina personalizada, donde México tiene enorme oportunidad de participar con innovación y con aplicaciones concretas (Galindo 2012).

Pero, explica, las ventajas de las ciencias genómicas sólo podrán estar al alcance de México si "México resolviera este tipo de retos [...] comenzando por la inversión en ciencia y tecnología que es la más baja dentro de los países de la OCDE sino de la inversión dentro de las economías emergentes grupo al que pertenece México” (Galindo 2012). Bertha, pregunta ¿Estamos muy lejos? Gerardo Jiménez responde:

\footnotetext{
México tiene un avance muy importante en la parte de investigación en ciencias genómicas, en instituciones, en recursos humanos, contribuciones científicas, incluso de reconocimiento internacional por sus avances en la genómica. En donde me parece que es fundamental invertir un esfuerzo especial es en la vinculación de llevar este conocimiento de laboratorio o de los artículos científicos que se generan a la generación de bienes y servicios que contribuyen al desarrollo económico [...]. No veo por qué México no podría lograrlo, me parece una cuestión de decisiones de alto nivel, de decisiones estratégicas y de que los tomadores de decisiones puedan conocer la importancia de la innovación en ciencia y
}

${ }^{3}$ El video fue realizado con base en un artículo científico (Moreno-Estrada et al. 2014). 
tecnología para alcanzar un beneficio y una prosperidad económica sustentable para México (Galindo 2012).

Posteriormete se daría la presentación oficial de la organización "Genómica y Bioeconomía" (Genómica y Bioeconomía 2012). Esta organización está conformada por "miembros destacados (de la sociedad mexicana) interesados en que México pueda usar la genómica como un puente rápido para la economía del conocimiento" (Galindo 2012). Desde entonces, esta organización impulsaría la discusión sobre la bioeconomía como posibilidad y oportunidad para el desarrollo económico de México. A la fecha, el sitio de esta organización hospeda una creciente colección de documentos técnicos y artículos. También aloja los videos de varios de los foros organizados en los últimos años. Es importante mencionar que entre los miembros directivos de esta organización se encuentran personas que también participaron durante el proceso de fundación del INMEGEN y durante sus primeros años de servicio. Esta coincidencia nos habla de un grupo de personas que desde diferentes espacios está buscando que la bioeconomía y las ciencias genómicas se beneficien de las decisiones de instancias gubernamentales y económicas.

En el libro Genómica y bioeconomía. Ventana de oportunidad para el crecimiento económico de México publicado en el 2012 por El Colegio de México y El Colegio Nacional se presenta un plan e iniciativa para el desarrollo de la bioeconomía en México. Pero a diferencia de otros documentos, este lo expone de manera comparativa. En este libro se expone el potencial de la bioeconomía en otras economías emergentes como China y Brasil. Se afirma que hay problemas en común (ambientales, desigualdad social, tráfico de drogas, armas, formas de exclusión y discriminación) y que estos problemas requieren de "innovaciones sociales e institucionales". La importancia de la bioeconomía está en que estas innovaciones sociales sólo pueden perdurar y ser eficientes si dependen de "un nuevo sistema de producción”.

\begin{abstract}
Asistimos a los efectos no deseados de un modelo de desarrollo hoy a todas luces insostenible que dio lugar a la contaminación ambiental, el cambio climático, el agotamiento de las fuentes de energía y la creciente destrucción de la capa de ozono, así como a fenómenos sociales igualmente perversos, como el aumento de la inseguridad, la pobreza, la desigualdad, el tráfico de drogas, armas y seres humanos, y formas inéditas de exclusión y discriminación. Si bien muchos de estos problemas requieren a su vez de innovaciones sociales e institucionales, éstas sólo pueden fundarse sobre un nuevo sistema de producción y desarrollo económico (Jiménez Sánchez et al. 2012, pp. 30-1).
\end{abstract}

Se señala que hay un falso dilema en creer que la inversión en los problemas sociales y ambientales goza de urgencia mayor que la inversión en ciencia y tecnología. Aunque parezca poco intuitivo, la inversión en ciencia y tecnología es la solución para los "grandes problemas nacionales".

Las economías de China, India y Brasil muestran un acelerado crecimiento en los últimos años, sin embargo, arrastran importantes rezagos sociales esencialmente relacionados con altos índices de pobreza, inequidad y contaminación, entre otros. No obstante, los gobiernos de estos países lograron superar el falso dilema de elegir entre la inversión para el desarrollo de innovaciones de frontera o para la solución de los grandes problemas nacionales. En los tres casos, la política de fomento a la ciencia y la tecnología establece un equilibrio entre ambos aspectos, con la certeza de que ninguna política social puede sostenerse en el tiempo sin un crecimiento paralelo y de que hoy día este crecimiento sólo puede ser alcanzado invirtiendo simultáneamente en áreas intensivas en conocimiento" [tecnológico y científico por supuesto, no de otro tipo] (Jiménez Sánchez et al. 2012, p. 54).

A finales del años siguiente en la inauguración del pasado foro internacional "Genómica, innovación y crecimiento económico. Integrando ciencia, tecnología e industria para un desarrollo sustentable" que tuvo lugar en la Ciudad de México del 25 al 27 de Noviembre 2013, el secretario general de la OCDE, el Dr. José Ángel Gurría, se dirigió a los asistentes con un mensaje en donde subrayó la importancia del foro que iba a comenzar y el rol de su organización frente al reto de lograr mejores vidas para 
todos. ${ }^{4}$ El video comienza con una animación de la cadena de DNA que brilla y se mueve de un lado a otro para dar paso al logo de Global Biotech Consulting Group, el sitio que hospeda este y otros videos. En el fondo azul que está detrás del secretario Gurría se lee "Better policies for better lives", que, como nos explica es el objetivo del trabajo de la OCDE.

\begin{abstract}
El proyecto del genoma humano abre posibilidades extraordinarias para el entendimiento de la salud humana y potencialmente ofrece curas para enfermedades que antes eran intratables [...] los efectos positivos son presenciados por la economía y el mercado de trabajo. Una evaluación reciente sugiere que desde 1998 la inversión del Gobierno de los Estados Unidos en el Proyecto del Genoma Humano ha producido una ganancia en la inversión cercana a 140 dólares por cada dólar gastado, y ha creado alrededor de 300 mil trabajos nuevos. La genómica y la biotecnología nos acercan a lograr sociedades sustentables y cumplir con las necesidades de las generaciones futuras. También juega un papel fundamental en afrontar los retos actuales como la población mundial que está por exceder los 9 mil millones para el 2050, una población que envejece rápidamente con un aumento en las necesidades de salud. Aumento en los requerimientos de recursos naturales que están en estado crítico debido al cambio climático. Una demanda creciente por alimentos y preocupaciones sobre seguridad alimentaria. Necesidades crecientes por la seguridad energética (2’44”).
\end{abstract}

Invertir en ciencia y tecnología y abrir paso a la bioeconomía representa hacer posibles las promesas de los mundos tecnosociales. No hacerlo implica perder la oportunidad de "sanar" la economía del país y "sanar" a los mexicanos. Las promesas de la bioeconomía traen de la mano por lo menos dos escenarios distópicos. El primero es aquel donde los problemas que se podrían resolver con la bioeconomía quedan sin resolver. El segundo es aquel dónde una nación extranjera se aprovecha de todos los beneficios de la bioeconomía (Goven \& Pavone 2015, p. 314). Promesas y distopías aparecen en el material arriba analizado.

El 30 de julio del 2014 se publicó en el Diario Oficial de la Federación el nuevo Programa Especial de Ciencia, Tecnología e Innovación (PECYT) 2014-2018 (DOF-Diario Oficial de la Federación 2014). En este programa se hace una proyección hasta el año 2038 donde México será "un actor global y destacado de la economía del conocimiento”. Para alcanzar este objetivo, el programa especifica los rubros de inversión, actores e instituciones que se deben fortalecer a lo largo de los diferentes sexenios. El PECYT ha sido diseñado bajo la convicción de que "la inversión en ciencia y tecnología es una herramienta fundamental para acceder a una economía de bienestar, basada en el conocimiento." El fin último del aumento en la producción y creación de "bienes y servicios de alto valor agregado" es mejorar los niveles de bienestar de los mexicanos. Por supuesto, una de las áreas en donde se debe fomentar la inversión es la biotecnología:

Fomentar las aplicaciones de la biotecnología para atender responsablemente las amenazas a la salud humana y animal, a la biodiversidad, a la disponibilidad de alimentos y de recursos energéticos, y a las provenientes del cambio climático. Se requieren incentivos y apoyos para lograr que las aplicaciones con organismos genéticamente modificados transiten adecuadamente por el entramado regulatorio (Diario Oficial de la Federación 2014).

\title{
4. Conclusión
}

En este artículo se mostró que la OCDE ha tenido una influencia importante en las decisiones sobre el desarrollo de la biotecnología y la bioeconomía en México. La OCDE, una organización que actúa como regulador y homogeneizador transnacional, posee medios de monitoreo que le permiten establecer prioridades, evaluaciones y estándares para los diferentes países miembros. A través de estos instrumentos de regulación es capaz de incidir en las decisiones que los diferentes países toman en relación a diversos aspectos de desarrollo humano y económico. Uno de los programas recientemente impulsado por esta organización es el de la bioeconomía como un nuevo modelo económico que

\footnotetext{
${ }^{4}$ El video de este mensaje y las demás conferencias del foro están alojadas en el sitio web de la firma consultora Global Biotech Consulting Group; una empresa que brinda servicios de asesoría para desarrollo de proyectos de biotecnología en diversas áreas, a empresas privadas, instituciones académicas e instancias gubernamentales en México y otros países(Global Biotech Consulting Group 2013).
} 
permitirá lidiar con las catástrofes humanas y ambientales que el mismo modelo capitalista ha generado. El proyecto bioeconómico implica la inversión en biotecnología para la creación de productos que ayuden a lidiar y resolver estos problemas. Promete un mundo tecnosocial donde la biotecnología repara el presente y garantiza el futuro del mismo modelo bioeconómico.

A partir del análisis de videos y diversos documentos se ha mostrado que la bioeconomía como proyecto económico ha ganado terreno en el contexto mexicano desde los años 2000 a la fecha. El INMEGEN es un buen ejemplo de las negociaciones que se han realizado en los últimos años. Justificar la construcción del INMEGEN con base en su efectividad para solventar los problemas de salud o la pertinencia de sus proyectos ha probado ser ineficiente. Como algunos autores han señalado, el INMEGEN fue aprobado sobre todo por representar una buena inversión económica. Sin embargo la historia no está completa si no se considera la influencia de la OCDE detrás de la construcción de este instituto. Sólo considerando el contexto transnacional donde la OCDE aparece como órgano regulador se puede entender con cabalidad la creación de este instituto. Así se puede ver que el INMEGEN es un medio más que un fin. Por esto la falta de resultados concretos para mejorar la vida de los mexicanos no ha perjudicado el funcionamiento o financiamiento de este instituto. Esto sugiere que en términos más generales, el proyecto bioeconómico no depende de ofrecer soluciones a los problemas que pretende resolver, sino de reproducir un modelo económico que permita la subsistencia del mismo sistema.

Bibliografía

American Express. Realize the potential (2011), recuperado de: https://www.facebook.com/video.php?v=10150147204406957\&set=vb.214208065504\&type=3\&video_source=p ages_video_set (acceso 13 enero 2015).

Brown, N. y M. Michael (2003), "A Sociology of Expectations: Retrospecting Prospects and Prospecting Retrospects", Technology Analysis $\mathcal{E}$ Strategic Management 15(1): 3-18.

Cooper, M. (2008), Life as Surplus. Biotechnology and Capitalism in the Neoliberal Era, Seattle: University of Washington Press.

Cruz Martínez, Á. (2013), "Invertir en ciencia es base del desarrollo económico, afirma Julio Frenk Mora", La Jornada, Sección "Sociedad y justicia" del 23 de febrero de 2013, recuperado de: http://www.jornada.unam.mx/2011/02/23/sociedad/045n2soc (acceso 14 de enero 2015).

Fortun, M. (2005), "For an Ethics of Promising, or: A Few Kind Words about James Watson”, New Genetics and Society 24(2): $157-73$.

Fortun, M. (2010), "Mediated Speculations in the Genomics Futures Markets", New Genetics and Society 20(2): 139. 156.

Galindo, B.A. (2012), "Entrevista al Dr. Gerardo Jiménez Sánchez", recuperado de: https://www.youtube.com/watch?v=iHeGRsiXuZE (acceso 1 de Noviembre 2014).

Genómica y Bioeconomía (2012), "Quienes somos", recuperado de: http://www.genomicaybioeconomia.org/quienes.html (acceso 1 de Noviembre 2014).

Global Biotech Consulting Group (2013), "GBC-Global Biotech Consulting Group", recuperado de: http://www.gbcbiotech.com/es/index.html (acceso 9 de febrero 2015).

Goven, J. y V. Pavone (2015), "The Bioeconomy as a Political Project: A Polanyian Analysis”, Science, Technology, $\mathcal{E}$ Human Values 40(3): 302-337.

Herrera Beltrán, C. (2009), "Presentaron ayer en Los Pinos mapa genómico de los mexicanos", La Jornada, Sección "Ciencias" del 12 de mayo de 2009, http://www.jornada.unam.mx/2009/05/12/ciencias/a02n1cie (acceso 9 de enero 2015). 
Jiménez Sánchez, G., Pozas, M.A., González, E.L., March, S., Zamalvide, J.M., Frenk, J. y G. Soberón (2012), Genómica y bioeconomía. Ventana de oportunidad para el crecimiento económico de México, México: El Colegio de México-El Colegio Nacional.

Kay, L.E. (1993), The Molecular Vision of Life. Caltech, The Rockefeller Foundation, and the Rise of the New Biology, New York: Oxford University Press.

López Beltrán, C. (ed.) (2011), Genes (E) Mestizos. Genómica y raza en la biomedicina mexicana, México: Universidad Nacional Autónoma de México.

López Beltrán, C. y V. García Deister (2013), "Scientific Approaches to the Mexican mestizo", História, Ciência, SaúdeManguinhos 20(2): 391-410.

López Beltrán, C. y F. Vergara Silva (2011), “Genómica Nacional: El INMEGEN y el Genoma del mestizo”, en López Beltrán, C. (ed.), Genes (E) Mestizos. Genómica y raza en la biomedicina mexicana, México: Universidad Nacional Autónoma de México, pp. 99-142.

Mahon, R. y S. McBride (2009), "Standardizing and Disseminating Knowledge: The Role of the OECD in Global Governance”, European Political Science Review 1(1): 83-101.

Mahon, R. y S. McBride (eds.) (2008), The OECD and Transnational Governance, Vancouver: The University of British Columbia Press.

Martínez, V. (15 de agosto de 2007), “(Agro) Espera Monsanto Duplicar Inversiones con Transgénicos”, Terra 15 de agosto de 2007, recuperado de: http://economia.terra.com.mx/noticias/noticia.aspx?idNoticia=200708150006_INF_522271 (acceso 20 de agosto 2015).

Montoya, M.J. (2011), Making the Mexican Diabetic. Race, Science and the Genetics of Inequality, California: University of California Press.

Moreno-Estrada, A., Gignoux, C.R., Fernández-López, J.C., Zakharia, F., Sikora, M., Contreras, A.V., Acuña-Alonzo, V., Sandoval, K., Eng, C., Romero-Hidalgo, S., Ortiz-Tello, P., Robles, V., Kenny, E.E., Nuño-Arana, I., BarqueraLozano, R., Macín-Pérez, G., Granados-Arriola, J., Huntsman, S., Galanter, J.M., Via, M., Ford, J.G., Chapela, R., Rodriguez-Cintron, W., Rodríguez-Santana, J.R., Romieu, I., Sienra-Monge, J.J., del Rio Navarro, B., London, S.J., Ruiz-Linares, A., Garcia-Herrera, R., Estrada, K., Hidalgo-Miranda, A., Jimenez-Sanchez, G., Carnevale, A., Soberón, X., Canizales-Quinteros, S., Rangel-Villalobos, H., Silva-Zolezzi, I., Burchard, E.G. y C.D. Bustamante (2014), "The genetics of Mexico recapitulates Native American substructure and affects biomedical traits", Science 344(6189): 1280-1285.

OECD (2009), The Bioeconomy to 2030. Designing a Policy Agenda: OECD.

OECD (2015a), History of the OECD, recuperado de: http://www.oecd.org/general/historyoftheoecd.htm (acceso 25 de Julio 2015).

OECD (2015b), OECD Week 2015: Investing in the future: People, Planet, Prosperity, recuperado de: https://www.youtube.com/watch?v=Y2QYeC4mQRM\#action=share (acceso 25 de Julio 2015).

OECD (2015c), OECD, recuperado de: About, http://www.oecd.org/about/ (acceso 25 de Julio 2015).

Pavone, V. (2012), “Ciencia, neoliberalismo y bioeconomía”, Revista CTS 7(20): 145-161.

Pérez, U.M. (2007), "Productores de 4 estados cultivarán maíz transgénico", La Jornada, Sección "Sociedad y Justicia" de 13 de noviembre de 2007, recuperado de: http://www.jornada.unam.mx/2007/11/13/index.php?section=sociedad $\&$ article=043n2soc (acceso 25 de Julio 2015).

DOF-Diario Oficial de la Federación (2014), Programa Especial de Ciencia, Tecnología e Innovación 2014-2018, México D.F.: Secretaría de Gobernación, recuperado de: http://www.dof.gob.mx/nota_detalle.php?codigo=5354626\&fecha=30/07/2014 (acceso 25 de Julio 2015).

DOF-Diario Oficial de la Federación (2011), Reglamento de la Ley de Producción, Certificación y Comercio de Semillas (2011), México D.F.: Secretaría de Gobernación, recuperado de: http://dof.gob.mx/nota_detalle.php?codigo=5207725\&fecha=02/09/2011 (acceso 25 de julio 2015).

Salzman, J. (2000), "Labor Rights, Globalization and Institutions: The Role and Influence of the Organization for Economic Cooperation and Development”, Michigan Journal of International Law (21): 769-848. 
Schwartz-Marín, E. (2011), "Protegiendo el "mextizaje”: INMEGEN y la construcción de la soberanía genómica", en López Beltrán, C. (ed.), Genes (E) Mestizos. Genómica y raza en la biomedicina mexicana, México: Universidad Nacional Autónoma de México, pp. 155-184.

Silva-Zolezzi, I., Hidalgo-Miranda, A., Estrada-Gil, J., Fernández-López, J.C., Uribe-Figueroa, L., Contreras, A., BalamOrtiz, E., del Bosque-Plata, L., Velázquez-Fernández, D., Lara, C., Goya, R., Hernández-Lemus, E., Dávila, C., Barrientos, E., March, S. y G. Jiménez-Sánchez (2009), "Analysis of genomic diversity in Mexican Mestizo populations to develop genomic medicine in Mexico", Proceedings of the National Academy of Sciences of the United States of America 106 (21): 8611-8616.

Taylor-Alexander, S. y E. Schwartz-Marín (2013), "Bioprophecy and the politics of the present: notes on the establishment of Mexico's national genomics institute (INMEGEN)”, New Genetics and Society 32(4): 333-349.

Villacorta S.R. (2009), Video Inductivo del INMEGEN, México D.F.: Patronato Fundador del INMEGEN, recuperado de: https://www.youtube.com/watch?v=PAFEH4gce7c (acceso 25 de Julio 2015).

Yoxen, E. (1982), “Giving Life a New Meaning: The Rise of the Molecular Biology Establishment”, en Elias, N., Martins, H. y R. Whitley (eds.), Scientific Establishments and Hierarchies, Dordrecht: Reidel, pp. 123-143.

Yoxen, E. (1985), “Speaking out about Competition. An Essay on 'The Double Helix" as Popularisation”, en Shinn, T. y R. Whitley (eds.), Expository Science: Forms and Functions of Popularisation, Dordrecht: Reidel, pp. 163-181. 